\title{
Research on Optimization for Units Start During Power System Restoration
}

\author{
Jun Chen ${ }^{1}$, Wei -xing Zhao ${ }^{1}$, Hua-ying Su${ }^{1}$, Jia-lin Bai ${ }^{1}$, Nian Liu ${ }^{2}$, Xiao-yan Qiu ${ }^{2}$, Qian Liao ${ }^{2}$ \\ ${ }^{1}$ Guizhou Power Grid Corporation of Dispatch Control Center, Guiyang, Guizhou, China \\ ${ }^{2}$ School of Electrical Engineering and Information Sichuan University, Chengdu, China \\ Email: ren_zeng@126.com,cd_qxy@sina.com
}

Received March, 2013

\begin{abstract}
Considering units starting and network constraints and the concept of optimization period, a optimization model which is a typical multi-constraint knapsack problem is established to solve the selection optimization problem of units starting in power system restoration period in this paper, and the objective of the model is to maximize the total power generation capability. A relative effectiveness assessment based on a improving data envelopment analysis is adopted to select the initial units to be started, genetic algorithms are employed to solve the knapsack problem to determine the most reasonable units be started at the current time. Finally, IEEE-39 bus system simulation result proves that the proposed model is feasible and effective.
\end{abstract}

Keywords: Power system; Units start; data envelopment analysis; knapsack problem

\section{Introduction}

In recent years, although modern power system has improved greatly in the running reliability, security, stability and economic, but large-scale blackout in the world still occur frequently, and the whole social activities and people's lives are impacted seriously [1-4]. Large-scale power system after the accident has usually affected scope, and power failure time is long, which put forward more severe challenges for the quick recovery of the accident of power system [5-7]. Therefore, how to make the system restored after the blackout becomes one of the important problems which each power grid have to faces. The system recovery process is usually divided into three stages, namely unit start-up, network reconfigurations and load recovery. Unit's recovery is the basis of the whole power system for the control of recovery. The key of unit's recovery is how to optimize the unit start according to the actual situation of the system during the recovery process. So, how to reasonably select the start units is the core issue of unit recovery.

The units start has been one of the important topics of the research field of power system. Many scholars have done a lot of research on the issue. The some of the concepts of power system faults recovery and the matters needing attention were concluded [8]. By using the ana-

\footnotetext{
*The project supported by GuiZhou Power Grid Corporation (12H0594), Technology Project of Science \& Technology Department of Sichuan Province (No.2011GZ0036)
}

lytical hierarchy process in the process of recovery unit start sorting, the recovery units and various factors of output were analyzed [9]. The genetic algorithm and the expert system were combined to agile develop units recovery strategy [10]. The data envelopment analysis method is adopted to assess the effectiveness of the recovery program technical verification; results included in the assessment system, and use the actual input, output index value to judge [11]. Therefore, considering units starting constraints and network constraints which include the unit start power constraint, maximum critical heat start time constraint, the minimum critical start time constraint and network constraints, a optimization model which is a typical multi-constraint knapsack problem is established to solve the selection optimization problem of units starting in power system restoration period in this paper, and the objective of the model is to maximize the total power generation capability. A relative effectiveness assessment based on a improving data envelopment analysis is adopted to select the initial units to be started, genetic algorithms is employed to solve the knapsack problem to determine the most reasonable units be started at the current time. Finally, IEEE-39 bus system simulation result proves that the proposed model is feasible and effective.

\section{Problem Fomulation}

\subsection{Objective Function}

The optimization goal to be maximize generation energy 
which is provided by units in the optimization period in this paper, so the objective function of the units start optimization model is defined as:

$$
\max \sum_{i=1}^{n} \int_{0}^{T} U_{i}(t)\left(P_{i}(t)-P_{c r, i}\right) d t
$$

where:

$n$ is the number of power plants; $T$ is the optimization period of the system unit recovery; $U_{i}(t)$ is the state of the $i(i=1, \ldots, n)$ unit ( 1 for start, 0 for outage; $P_{c r, i}$ is the required starting power of the $i$ unit in startup process; $P_{i}(t)$ is the output function of the $i$ unit, as shown in Figure 1.

\subsection{Constraints}

Unit constraint includes critical time constraints and starting power constraint.

$$
\begin{gathered}
0 \leq T_{s, i} \leq T_{C H, i} \\
T_{s, i} \geq T_{C C, i} \\
P_{0}\left(t_{0}\right)+\sum_{k=1}^{n} C_{k}(t)\left(P_{k}(t)-P_{c r, k}\right)-P_{c r, i} \geq 0
\end{gathered}
$$

where:

$T_{C H, i}$ is the maximum critical hot start time of $i$ hot start unit ; $T_{C C, i}$ is the minimum critical cold start time of $i$ cold start unit ; In the formula (4), $P_{0}\left(t_{0}\right)$ is the initial power of the system, Where the second term represents the system power which provided by grid units; $k$ is the number of grid unit; $C_{k}(t)$ is the state of the $k$ grid unit; $P_{k}(t)$ is the output function of the $k$ grid unit; $P_{c r, k}$ is the required starting power of the $k$ grid unit in startup process.

Meanwhile, network constraints were considered in this optimization model. The unit start network constraints can through the following indexes to show:

Power transmission path index (including power transmission path charging reactive power and voltage switching frequency).

Load importance of index (considering the relative importance of the units which is started near the load).

Hot start pressing index (mainly reflected in the current time step, the unit is near its biggest critical heat start time limit level).

\section{Solving Method}

\subsection{The Solution Strategy of Optimization Mod- el}

The units start is a more complicated optimization problem; the direct solution has the certain difficulty. Using model discrimination technique, the optimization time $T$ is divided into time step $N$, so the complicated continu- ous time problem is disserted to be a simple optimization problem of the unit start in the every step. If $h$ is step length, then each step for the start time and end time is respectively $h\left(k_{t}-1\right)$ and $h k_{t}\left(k_{t}=1, \ldots, N\right)$, according to the formula (1), the every step of objective function is

$$
\max \sum_{i=1}^{n} \int_{h\left(k_{t}-1\right)}^{T} U_{i}(t)\left(P_{i}(t)-P_{c r, i}\right) d t
$$

From the above model, units start optimization problems with multiple constraints is a knapsack problem. In this paper, the optimization problem was decomposed, a part of constraint conditions which were smaller correlation degree with objective function calculation were extracted in advance, and start unit were elected preliminarily. The optimization problem was slack for the problem only considering starting power constraint conditions, the unit can provide the biggest power generation. By solving one-dimensional constraint knapsack problem, the most reasonable current time start units were determined quantitatively.

\subsection{Primary Election of Start unit}

Data Envelopment Analysis (DEA) [12] is a method, on which based relative efficiency concept as the foundation, through the mathematical programming compare the relative efficiency of each decision making unit, and to make efficiency evaluation. The CCR model (in Charnes, Cooper, Rhodes and three authors initials named) and its equivalent linear programming model were commonly used to solve the multi-objective decision making problem.

When the CCR model is used to evaluate and compare, if multiple decision unit efficiency evaluation index $E_{k}$ are equal to 1 , the decision making units cannot be given comparison and ranking. An improved DEA model, that is, super efficiency DEA model $[13,14]$ is fully applied to compare and rank the decision making unit in this paper.

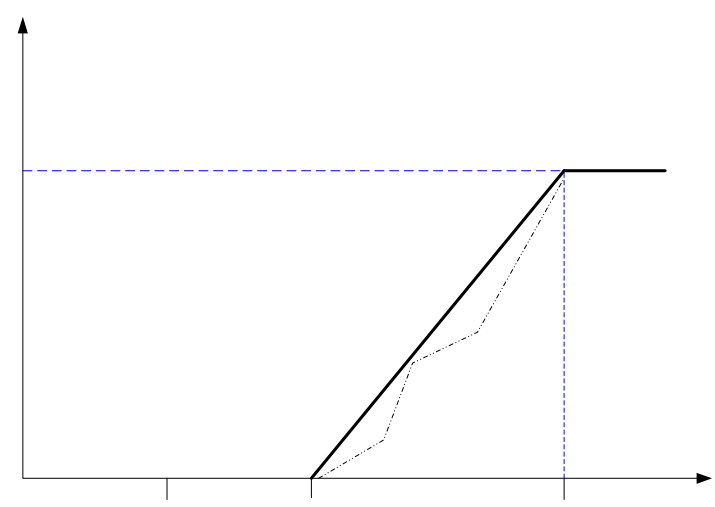

Figure 1. Schema of generation unit output function. 
In the units recovery model, according to start units selection principle, each optional start units are launched as decision unit, then combined with the improved DEA model, the input and output index of the unit relative efficiency evaluation are established. The index and its calculation method as are shown in Table $\mathbf{1}$.

The calculation method of each index interpretation is as follows.

Unit start power value is $P_{c r, i}$ value.

Power transmission path index value is every $\mathrm{km}$ line charging power $Q_{c j}$ multiply line length $L_{j}$ product plus weighted value of voltage conversion number $Z_{T}$.

Load importance index is the reciprocal of each unit near important load $L_{\text {urg }, i}$ ratio all load $L_{\Sigma, i}$.

Hot start pressing index is difference value of unit $T_{C H, I}$ and the current time step over time $h k_{t}$.

Unit power output index is the integral of the output of generating unit in the optimization of time period.

\section{Determining the most Reasonable Start Unit by Solving Knapsack Problem}

Units are primarily elected by the improved DEA, the original optimization problem is slack for a one dimensional constraint knapsack problem. In this problem, the "backpack volume", "efficiency indicators" and "volume index" corresponding to the total power of the start units which whole system is available for in the period, the generation energy of units in the optimization period and the starting power of the optional units, the model such as type (6) and (7) show.

$$
\begin{gathered}
\max \sum_{i=1}^{n} \int_{h\left(k_{t}-1\right)}^{T} U_{i}(t)\left(P_{i}(t)-P_{c r, i}\right) d t \\
P_{0}\left(t_{0}\right)+\sum_{k=1}^{n} C_{k}(t)\left(P_{k}(t)-P_{c r, k}\right)-P_{c r, i} \geq 0
\end{gathered}
$$

Genetic algorithm is the most representative of the evolutionary algorithm, which is the most basic intelligent optimization algorithm; this method has the parallel search, group optimization characteristics, widely used to solve various completely non-deterministic polynomial time problems. Because the quantity of units after primary selection is not big, the most reasonable units are deter- mined by solving knapsack problem using genetic algo- rithm.

\section{Case Study}

The IEEE-39 node system [15] is adopted to analyze this problem, the system topology structure shown in Figure 2. And assume the initial conditions: the unit $30-1$ as black start unit, $t_{0}=1.5 \mathrm{~h}, T=12 \mathrm{~h}, N=48$. Each unit parameter is shown in Table 2.
Table 1. Input indices and output indices used in assessmentof units.

\begin{tabular}{ccc}
\hline Index & Details & Calculation method \\
\hline A & Unit start power & $P_{c r, i}$ \\
B & Power transmission path & $Q_{c j} L_{j}+Z_{T}$ \\
C & Load importance & $L_{\Sigma, i} / L_{u r g, i}$ \\
D & Hot start pressing & $T_{C H, I-} h k_{t}$ \\
E & Unit power output & $\int_{h\left(k_{i}-1\right)}^{T} U_{i}(t)\left(P_{i}(t)-P_{c r, i}\right) d t$ \\
\hline
\end{tabular}

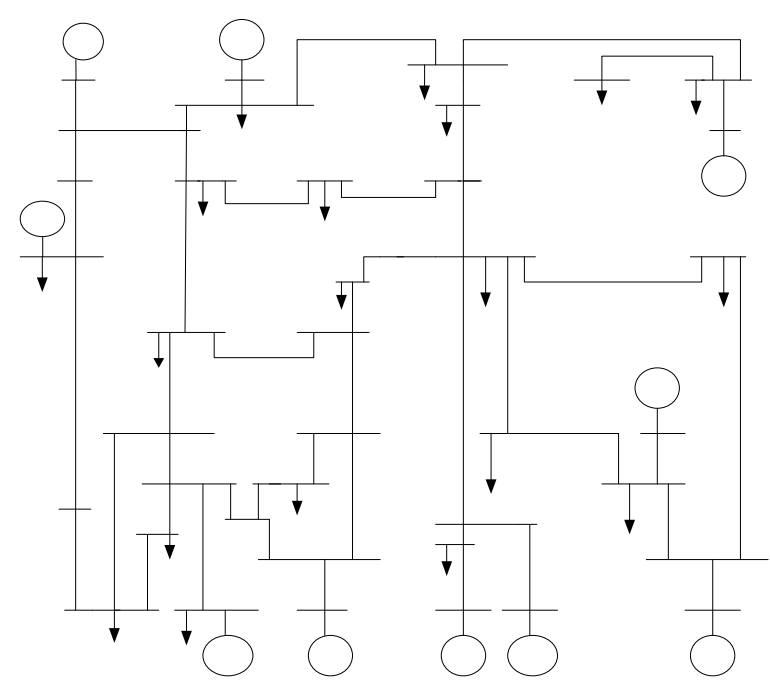

Figure 2. Structure chart of IEEE- 39-bus power system.

Table 2. Parameters of the units of IEEE- 39-bus power

\begin{tabular}{|c|c|c|c|c|c|c|c|}
\hline Node & Unit & $\begin{array}{l}P_{c r} l \\
\text { MW }\end{array}$ & $\begin{array}{l}P_{M} / \\
\text { MW }\end{array}$ & $\begin{array}{c}K_{\mathrm{p}} / \\
\mathrm{MW} / \mathrm{h}\end{array}$ & $\begin{array}{c}T_{s, i}{ }^{\prime} \\
/ \mathrm{h}\end{array}$ & $\underset{\mathrm{h}}{T_{C C, i} /}$ & $\begin{array}{c}T_{C H, i} / \\
\mathrm{h}\end{array}$ \\
\hline 30 & $30-1$ & 0 & 250 & 166.67 & 0 & N/A & N/A \\
\hline \multirow{2}{*}{31} & $31-1$ & 18 & 378 & 150 & 0.7 & 3 & N/A \\
\hline & $31-2$ & 15 & 300 & 120 & 0.5 & N/A & 3 \\
\hline \multirow{2}{*}{32} & $32-1$ & 17.5 & 350 & 130 & 0.67 & N/A & 3.5 \\
\hline & $32-2$ & 15 & 300 & 120 & 0.5 & N/A & 3 \\
\hline \multirow{2}{*}{33} & $33-1$ & 15 & 332 & 108 & 0.6 & 2.3 & N/A \\
\hline & $33-2$ & 15 & 300 & 120 & 0.5 & $\mathrm{~N} / \mathrm{A}$ & 2 \\
\hline \multirow{3}{*}{34} & $34-1$ & 16.5 & 308 & 100 & 1.1 & $\mathrm{~N} / \mathrm{A}$ & 4.2 \\
\hline & $34-2$ & 15 & 250 & 100 & 1.1 & N/A & 3.5 \\
\hline & $35-1$ & 17.5 & 350 & 130 & 0.67 & N/A & 3.5 \\
\hline \multirow[t]{2}{*}{35} & $35-2$ & 8 & 150 & 90 & 0.83 & 2 & N/A \\
\hline & $35-3$ & 8 & 150 & 90 & 0.83 & 2 & N/A \\
\hline \multirow{2}{*}{36} & $36-1$ & 10 & 200 & 120 & 0.5 & N/A & 2 \\
\hline & $36-2$ & 18 & 350 & 130 & 0.67 & N/A & 3.5 \\
\hline \multirow{2}{*}{37} & $37-1$ & 10 & 200 & 120 & 0.5 & N/A & 2 \\
\hline & $37-2$ & 17.5 & 350 & 130 & 0.67 & N/A & 3.5 \\
\hline \multirow{3}{*}{38} & $38-1$ & 10 & 200 & 120 & 0.5 & N/A & 2 \\
\hline & $38-2$ & 10 & 200 & 120 & 0.5 & N/A & 2 \\
\hline & $38-3$ & 20 & 430 & 130 & 1.2 & N/A & 3 \\
\hline \multirow{2}{*}{39} & $39-1$ & 22 & 500 & 200 & 1.2 & N/A & 4 \\
\hline & $39-2$ & 22 & 500 & 200 & 1.2 & N/A & 4 \\
\hline
\end{tabular}
system. 


\section{Results}

In the recovery process of the first step for $k_{t}=1$, unit $31-1,33-1,35-2$ and 35-3 are not meet the minimum critical time limit, so those are units unable to cold start, the rest of the 16 units can satisfy the start condition. The first, the optional units are viewed as decision making units to evaluate its relative efficiency, the corresponding input and output indices such as shown in Table 3.

According to the input and output indices, the improved DEA model is established. Through the linear programming to solve, the efficiency Ek of the corresponding each unit is confirmed by using the linear programming to solve, such as shown in Table 4.

According to the definition of efficiency evaluation, the unit 35-1 , 36-1, 37-1, 38-1, 38-2 and 39-2 are relatively effective, this six units can be used as the feasible solution for the first step. With feasible solution as the foundation, combined with optimization process and the corresponding solving knapsack problem, the unit 36-1, $37-1,38-1$ and $38-2$ are finally determined to be the optimal solution, which are started firstly in the current time step. By the same way, the optimization start units can be gradually determined in each subsequent time step, such as shown in Table 5.

Table 3. Input and output indices of selecting units.

\begin{tabular}{|c|c|c|c|c|c|}
\hline \multirow{2}{*}{ Unit } & \multicolumn{4}{|c|}{ Input index } & \multirow{2}{*}{$\frac{\text { Output index }}{E}$} \\
\hline & A & B & $\mathrm{C}$ & $\mathrm{D}$ & \\
\hline $31-1$ & 18 & 56.35 & 6 & 5 & 3365.8 \\
\hline $31-2$ & 15 & 56.35 & 3.4 & 0.5 & 2839.4 \\
\hline $32-1$ & 17.5 & 33.5 & 6.5 & 5 & 3298.1 \\
\hline $32-2$ & 15 & 33.5 & 7.55 & 3.5 & 2792.5 \\
\hline $33-1$ & 15 & 45.76 & 7.6 & 5 & 2983.7 \\
\hline $33-2$ & 15 & 45.76 & 9 & 1.55 & 2865.3 \\
\hline $34-1$ & 16.5 & 37.26 & 5.6 & 5 & 2870.0 \\
\hline $34-2$ & 15 & 37.26 & 8.67 & 3.5 & 2366.4 \\
\hline $35-1$ & 17.5 & 50.7 & 15 & 3.5 & 3285.9 \\
\hline $35-2$ & 8 & 50.7 & 4 & 0.8 & 1452.5 \\
\hline $35-3$ & 8 & 50.7 & 7.57 & 5 & 1452.5 \\
\hline $36-1$ & 10 & 29.65 & 25 & 5 & 1927.8 \\
\hline $36-2$ & 18 & 29.65 & 4.8 & 5 & 3312.7 \\
\hline $37-1$ & 10 & 30.2 & 22.5 & 5 & 1925.0 \\
\hline $37-2$ & 17.5 & 30.2 & 7.33 & 3.5 & 3294.2 \\
\hline $38-1$ & 10 & 26.45 & 10 & 5 & 1928.5 \\
\hline $38-2$ & 10 & 26.45 & 17.56 & 1.55 & 1928.5 \\
\hline $38-3$ & 20 & 34.6 & 8.9 & 5 & 4129.1 \\
\hline $39-1$ & 22 & 34.6 & 6.5 & 5 & 4752.4 \\
\hline $39-2$ & 22 & 34.6 & 10.35 & 5 & 4752.4 \\
\hline
\end{tabular}

Table 4. The effective index of units.

\begin{tabular}{cccccccc}
\hline Unit & $31-1$ & $31-2$ & $32-1$ & $32-2$ & $33-1$ & $33-2$ & $34-1$ \\
\hline$E_{k}$ & 0.845 & 0.975 & 0.983 & 0.950 & 0.942 & 0.961 & 0.969 \\
Unit & $34-2$ & $35-1$ & $35-2$ & $35-3$ & $36-1$ & $36-2$ & $37-1$ \\
$E_{k}$ & 0.946 & 1 & 0.868 & 8.837 & 1 & 0.953 & 1 \\
Unit & $37-2$ & $38-1$ & $38-2$ & $38-3$ & $39-1$ & $39-2$ & N/A \\
$E_{k}$ & 0.976 & 1 & 1 & 0.853 & 0.981 & 1 & N/A \\
\hline
\end{tabular}

Table 5. Units start result of each time-step.

\begin{tabular}{ll}
\hline$k_{t}$ & \multicolumn{1}{c}{ The priority start units } \\
\hline 1 & $36-1$ 37-1 38-1 38-2 \\
2 & $35-139-1$ \\
3 & $37-2$ 39-2 \\
4 & $31-2,32-1,32-2,33-2,34-1$ \\
5 & $33-1,34-2$ 36-2 38-1 \\
6 & N/A \\
7 & N/A \\
8 & $35-2,35-3$ \\
9 & N/A \\
10 & $31-1$ \\
\hline
\end{tabular}

\section{Conclusions}

The optimization strategy of the unit start was researched in this work. An optimization model is established to solve the selection optimization problem of units start in power system restoration, and the objective of the model is to maximize the total power generation. According to the optimization model for a typical with multiple constraints of knapsack problem, a relative effectiveness assessment based on a improving data envelopment analysis is adopted to select the initial units to be started, and genetic algorithm is employed to solve the knapsack problem to determine the most reasonable units be started at the current time. Finally, through a example of system simulation, the result proves that the proposed method is feasible and effective.

\section{REFERENCES}

[1] US-Canada Power Outage Task Force. Final Report on the August 14th 2003 Blackout in the United States and Canada [EB / OL]. http://www.ferc.gov.

[2] G. Andersson, P. Donalek and R. Farmer, et al., "Causes of the 2003 Major Grid Blackouts in North America and Europe, and Recommended Means to Improve System 
Dynamic Performance. IEEE Transactions on Power Systems," Vol. 20, No. 4, 2005, pp. 1922-1928. doi:10.1109/TPWRS.2005.857942

[3] Y. V. Makarov, V. I. Reshetov, A. Stroev, et al., "Blackout Prevention in the United States, Europe, and Russia," Proceedings of the IEEE, Vol. 93, No. 11, 2005, pp. 1942-1955.doi:10.1109/JPROC.2005.857486

[4] X. Y. Chen, Y. P. Chen, C. Y. Li, et al., "Constructing Wide-area Security Defensive System in Bulk Power Grid_ A Pondering over the Large-scale Blackout in the European Power Grid," Automation of Electric Power Systems, Vol. 31, No. 1, 2007, pp. 4-8.

[5] Cascade to Black [system blackouts]. IEEE Power and Energy Magazine, Vol. 2, No. 3, 2004, pp. 54-57. doi:10.1109/MPAE.2004.1293601

[6] S. H. Horowitz and A. G. Phadke, "Boosting Immunity to Blackouts, "Power and Energy Magazine," Vol. 1, No. 5, 2003, pp. 47-53. doi:10.1109/MPAE.2003.1231691

[7] Y. Kojima. S. Warashina, M. Kato and H. Watanabe, "Application of Knowledge Engineering Techniques to Electric Power System Restoration,” in Proc. 1988 IEEE Artifkiul Intelligence Jar lndiis/riul Applicat ions. M'orkshop on, pp. $320-325$.

[8] J. Hu, Z. Z. Guo, Y. C. Liu and Q. Y. Guo, "Power System Restoration And Analysis Of Restoration Sequence of Generating Sets," Power System Technology, Vol. 28, No. 18, 2004, pp. 1-4.

[9] Z. Z. Dong, J. L. Jiao, Q. H. Sun, “Arrangement of Prior- ity Sequence of Thermal Unit Restoration on Analytical Hierarchy Process Model," Power System Technology, Vol. 21, No. 6, 1997, pp. 48-51, 54.

[10] K. Salek, U. Spanel and G. Krost, "Flexible Support for Operators in Restoring Bulk Power Systems," CIGRE/IEEE PES International Symposium on Quality and Security of Electric Power Delivery Systems, Montreal, Canada, Oct. 2003, pp. 187-192.

[11] Y. Liu, X. P. Gu, D. Zhang, "Data Envelopment Analysis Based Relative Effectiveness Assessment of Power System Black-start Plans," Proceedings of the CSEE, Vol. 26, No. 5, 2006, pp. 32-37, 94.

[12] Q. Liu, L. B. Shi, M. Zhou, G. Y. Li and Y. X. Ni, "Optimal Strategy for Units Start-up During Power System Restoration," Transactions of China Electrotechnical Society, Vol. 24, No. 3, 2009, pp. 164-170.

[13] Y. Chen, Ranking Efficient Units in DEA," OMEGA, Vol. 32, No. 6, 2004, pp. 213-219.

[14] X. Mei, P. T. Harker, "Note: Ranking DMUs with Infeasible Super-efficiency DEA Models," Management Science, Vol. 48, No. 5, 2002, pp. 705-710. doi: $10.1287 / \mathrm{mnsc} .48 .5 .705 .7805$

[15] X. Gu and H. Zhong, "Optimisation of Network Reconfiguration Based on a Two-layer Unit-restarting Framework for Power System restoration," IET Generation, Transmission \& Distribution, Vol. 6, No. 7, 2012, pp. 693-700. doi:10.1049/iet-gtd.2011.0591 